\section{Insights into E3805: the CHAARTED trial}

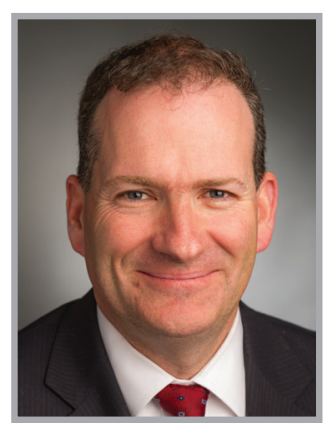

Christopher J Sweeney* speaks to Dominic Chamberlain, Commissioning Editor: A Medical Oncologist at the Dana-Farber Cancer Institute (MA, USA), Christopher J Sweeney is a member of the American Society of Clinical Oncology, the Eastern Cooperative Oncology Group-ACRIN, Alliance and the American Association for Cancer Research. In 1992, Sweeney received his medical degree from the University of Adelaide (Australia) and completed an internship at the Royal Adelaide Hospital (Australia). Dr Sweeney did his residency in internal medicine at Gundersen Lutheran Medical Center (WI, USA) and a fellowship in hematology/oncology at Indiana University Medical Center (IN, USA), where he was later appointed Associate Director for Clinical Research for the Simon Cancer Center. Dr Sweeney joined the Lank Center for Genitourinary Oncology at Dana-Farber Cancer Institute and Harvard Medical School (MA, USA) in 2009. His primary research interest is drug discovery and development. His academic focus is primarily on the management of genitourinary malignancies, with a focus on prostate and testicular cancer.

\section{Q What sparked your interest in cancer research?}

In short, I got into cancer research to help people who were very vulnerable and really needed help. When I was doing my residency and I came across people with cancer, I realized that we had really not perfected the treatment and wanted to get involved to move the ball forward.

\section{Q What led you to your current interest in genitourinary oncology?}

When I was at Indiana University (IN, USA) they had a very active Phase I clinical trials unit and this allowed me to take part in drug development, and there I also began to focus on prostate cancer. I worked on other cancers but most of my work involved prostate cancer. My interest comes from the fact that a lot of men, too many men, die from prostate cancer and although there is a lot of work concerning screening and finding the cancer so it can be treated early, still too many men are dying. I believe we need to also put the spotlight on advanced disease before it becomes hormone refractory and make our cancer therapies more effective.

\footnotetext{
*Dana-Farber Cancer Institute, MA, USA; Harvard Medical School, MA, USA; Christopher_Sweeney@dfci.harvard.edu
}

\section{KEYWORDS}

- androgen-deprivation therapy

- docetaxel • hormone-sensitive metastatic prostate cancer

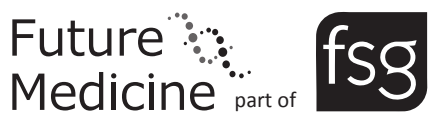


Q You recently presented results of a Phase III trial on a combination of androgen deprivation therapy \& docetaxel in prostate cancer. Can you tell us a little about what led to this trial and what is planned next?

We do a lot of our research in the last phase of prostate cancer where the patient's cancer has progressed to the bone and other organs while on androgen deprivation therapy (known as castration-resistant prostate cancer). There is a lot of drug development in this setting with each new therapy resulting in on average couple of months of improvement in the overall survival. One of the questions is: we have drugs that are clearly active and have a benefit in advanced castration-resistant disease, so what if we take those drugs, or one of those drugs, to the setting where patients are starting their hormone treatment? The reason is a lot of drugs are developed in the castration-resistant setting because it is relatively quick to get a readout on whether your drug works and then the regulatory bodies such as the US FDA can say: "we see that improvement and so we will approve it," whereas when you do studies in hormone-sensitive diseases like the CHAARTED trial it takes a longer time to get a readout on whether treatment $A$ versus treatment B works. This particular study was conducted by the National Cancer Institute. Sanofi provided the drugs, and it was a nice collaboration because back in 2005 Sanofi recognized the importance of the trial and so worked with the ECOG team outside the drug development 'timeline' within their patent lifespan, as the drug would be generic when the study finished.

Q What effects do you think this research will have on the clinic?

All interests declared, I wrote the study and spent many years on it, so that does perhaps need to be factored into what I say. However, a lot of people have told me (I do not think they are just trying to be nice to me) that it will change their practice - with a special emphasis on men with high volume of metastases - that instead of waiting for the cancer to grow after having been on testosterone suppression and having castration-resistant disease, they will use it early. We normally talk about 2-3 months improvement in survival but for the whole of this group the improvement in survival was 13 months, which is a big improvement in overall survival and something we rarely see in oncology and it was 17 months benefit in those with high-volume disease.
Q What other projects are you involved in at the moment?

Well I am trying to train to ride a 200-mile bike ride, but that probably is not what you are interested in! The follow-on question from the above study was that this was a big improvement in outcome, so why did it happen? I do not think the chemotherapy made the hormone therapy more effective, I think the hormone therapy made the chemotherapy very effective and we know that hormones plus radiation are much more effective than hormones alone or radiation alone. Recent work has shown that hormone treatment (suppression of testosterone) decreases cellular processes that act to help with DNA repair, thus making cells more vulnerable to radiation treatment. The question I am trying to work out in the laboratory is as follows: if we are seeing this for radiation, can we see something similar for cancer chemotherapy? With the hormone treatment do we inhibit genes, or proteins or something that makes the cancer cells more susceptible to chemotherapy? The fact is that we do not know, so we are trying to understand. Our hypothesis is that the hormone treatment reduces a cellular process that makes the cancer cells otherwise resistant to treatment.

\section{Q What do you feel is the biggest challenge facing prostate cancer treatment today?}

A lot of people are diagnosed with prostate cancer early, and a lot of research is assessing what makes a cancer that is diagnosed early one that needs to be treated with surgery or radiation when localized, so one question is: "how do we prevent overtreatment?" that is a big challenge. However, my personal focus is on cancer that has spread to the bone, to the lungs and other parts of the body - a stage of cancer that carries a high chance of leading to a patient's death. Therefore, my area of research is how to improve therapy for disseminated cancer and I think the biggest challenge is we still have a large number of men dying from cancer that has spread to the bone, despite treating many men with localized disease (including many men who did not need surgery or radiation treatment).

\section{Q How would you tackle this?}

I think we need to try and understand what drives the underlying cause of resistance. Hormone treatment is an effective treatment, many men live for years with it, and some have a response that lasts 6 months, some 6 years. 
What we need to understand is how we can have more men receive a treatment that works for a longer time. The other thing to consider is that nearly all men do not want to have their testosterone suppressed and receive the side-effect profile from doing that, so the question is: "can we work out how to make prostate cancer respond without all of the collateral effects of long-term testosterone suppression?"

\section{Q Where do you see the field in the next 10 years?}

It would be great to identify the genes that make cells resistant to chemotherapy and block those genes so that we can have a quick period of treatment such as six cycles every 3 weeks of a treatment. We could then stop and wait for the patients to recover, meaning that the patients would not have to be on hormone treatments that suppress their testosterone for a long time. Give the chemotherapy, block the genes and hopefully make the cancer treatment much more successful, having people live longer and if we can find key cancer cell vulnerabilities, eventually eradicate all disseminated cancer.

In addition, if we learn from history, 10 years ago docetaxel was approved for castration-resistant disease and was improving survival for a few months. If we fast forward 10 years, we use the drug early and now improve survival, as seen in our previously mentioned study, increase median survival by about 17 months in patients with high volume disease. If we can confirm this in the UK STAMPEDE trial and everyone be convinced about its benefits and further understand the key drivers of cancer and develop new therapies, we will prolong survival for an even longer period of time. More specifically, we need to know the drivers and inhibit those drivers specifically without having to resort to toxic treatments. This means inhibiting those drivers without all the collateral damage such as the increased risk of infections, losing hair, irritating nerves and so on. I think in the next 10 years it is quite possible that we will know those genes, know the drivers and be able to treat cancer much more efficiently.

\section{Q If you had unlimited resources, what would you do \& why?}

That is a great question. What I can say is that there are a lot of resources being pooled into interrogating cancers with some great technologies that are out there. It is hard to say what I would do because whenever I want to do something I always consider how it can be done in a cost-effective manner. It is important not to view resources as just including money; a very precious resource are the patients who volunteer for a trial as well. If resources were unlimited I would do what I am doing but, on a larger scale, evaluating all the new drugs active in a castration-resistant setting, and use them earlier in the disease setting - such as hormone sensitive metastatic disease and adjuvant to high-risk localized curable disease - and collect specimens to find out why patients are not being cured. So I would continue to do the trials in earlier, more sensitive prostate cancer and continue to look at the cancer cells and work out why those that aren't being eradicated become resistant. One of my catchphrases is: "treating castration-resistant disease is a little bit defeatist (because it is already become resistant), let us try and start treatment early when they are more at risk of being cured - let us go forward by moving backward."

Q Finally, what would your message to the oncology community be?

Commitment to the clinical trial process is essential. We will not make advances unless we do trials of treatment $\mathrm{A}$ versus treatment $\mathrm{B}$. As clinicians come up with questions on the front line, collaboration with the academic researchers - laboratory-based and clinical trialists - is vital to work out ways to address the questions and hopefully move the ball forward. Commitment to the clinical trial process needs to start from the primary care provider to the urologist and radiation oncologist to the medical oncologist; it is a team sport and we all need to be in it together.

\section{Disclaimer}

The opinions expressed in this interview are those of the interviewee and do not necessarily reflect the views of Future Medicine Ltd

\section{Financial \& competing interests disclosure}

CJ Sweeney has carried out consulting with compensation for Sanofi, BIND, Janssen, Astellas, Genentech, Bayer. CJ Sweeney has no other relevant affiliations orfinancial involvement with any organization or entity with a financial interest in or financial conflict with the subject matter or materials discussed in the manuscript apart from those disclosed.

No writing assistance was utilized in the production of this manuscript. 